\title{
Gene and isoform expression signatures associated with tumor stage in kidney renal clear cell carcinoma
}

\author{
Qi Liu ${ }^{1,2+}$, Shilin Zhao ${ }^{1 \dagger}$, Pei-Fang Su${ }^{3}$, Shyr Yu ${ }^{1,4,5^{*}}$ \\ From The International Conference on Intelligent Biology and Medicine (ICIBM 2013) \\ Nashville, TN, USA. 11-13 August 2013
}

\begin{abstract}
Background: Identification of expression alternations between early and late stage cancers is helpful for understanding cancer development and progression. Much research has been done focusing on stage-dependent gene expression profiles. In contrast, relatively fewer studies on isoform expression profiles have been performed due to the difficulty of quantification and noisy splicing. Here we conducted both gene- and isoform-level analysis on RNA-seq data of 234 stage I and 81 stage IV kidney renal clear cell carcinoma patients, aiming to uncover the stage-dependent expression signatures and investigate the advantage of isoform expression profiling for identifying advanced stage cancers and predicting clinical outcome.

Results: Both gene and isoform expression signatures are useful for distinguishing cancer stages. They provide common and unique information associated with cancer progression and metastasis. Combining gene and isoform signatures even improves the classification performance and reveals additional important biological processes, such as angiogenesis and TGF-beta signaling pathway. Moreover, expression abundance of a number of genes and isoforms is predictive of the risk of cancer death in an independent dataset, such as gene and isoform expression of ITPKA, the expression of a functional important isoform of UPS19.
\end{abstract}

Conclusion: Isoform expression profiling provides unique and important information which cannot be detected by gene expression profiles. Combining gene and isoform expression signatures helps to identify advanced stage cancers, predict clinical outcome, and present a comprehensive view of cancer development and progression.

\section{Background}

Stepwise progression of cancer malignancy has been clinically well defined [1]. In the early stage, the cancer cells, confined to a very limited area, are not invasive and metastatic, whereas in the late stage, the cells, spreading to distant sites in the body, are highly invasive and metastatic. Comparative analysis of genetic, epigenetic, and expression alterations between early and late stage cancers can help to understand cancer progression and metastasis mechanisms and predict the clinical aggressiveness of cancer [1]. Many studies have been extensively performed on various types of human

\footnotetext{
* Correspondence: yu.shyr@vanderbilt.edu

+ Contributed equally

'Center for Quantitative Sciences, Vanderbilt University School of Medicine, Nashville, TN 37232, USA

Full list of author information is available at the end of the article
}

cancers [2-22]. For example, molecular mutations were reported to be accumulated in a fashion that paralleled the clinical progression of colorectal cancer $[5,7,10]$. Changes in DNA methylation were also found to be cumulative with disease progression in ovarian cancer, gastric cancer and prostate cancer [3,8,11]. Stage-dependent mRNA and microRNA expressions were identified in neuroblastoma, colon cancer, bladder cancer and gastric cancer $[2,4,6,9]$. Based on these discovered genetic, epigenetic, and expression alternations, models of tumor progression have been constructed, and the process of tumor progression and metastasis has been studied.

In addition to genetic, epigenetic, and expression alternations, post-transcriptional deregulation also plays an important role in cancer progression [17-23]. For example, alternative splicing of FGFR1 was found to be associated with tumor stage and grade; isoform switch 
of FGFR1 may result in a proliferative advantage that plays a key role during bladder tumor progression [18]. Alternative splicing leads to expression changes of specific isoforms, possibly without overall mRNA expression alternations. Isoform expression alternations, however, have not been widely studied partly due to the difficulty of isoform expression quantification. Recently, RNA-seq has been increasingly used to discover and profile the whole transcriptome [24]. The digital nature of RNAseq technology coupled with powerful bioinformatics methods including Alexa-seq [25], IsoEM [26], Multisplice [27], MISO [28], Cufflinks [29,30], iReckon [31] and RSEM [32,33], which aim to quantify isoform expression accurately, provides the opportunity of systematically studying expression alternations at isoform level. However, due to the complexity of transcriptome and read assignment uncertainty, calculating isoform abundance from incomplete and noisy RNA-seq data is still challenging [34]. The advantage of using isoform expression profiles to identify advanced stage cancers and predict clinically aggressive cancers remains unclear.

In this study, we performed a comprehensive analysis on RNA-seq data of 234 stage I and 81 stage IV kidney renal clear cell carcinoma (KIRC) patients. We identified stage-dependent gene and isoform expression signatures and quantitatively compared these two kinds of signatures in terms of cancer stage classification, biological relevance with cancer progression and metastasis, and independent clinical outcome prediction. We found that isoform expression profiling provided unique and important information that could not be detected at the gene level. Combining isoform and gene signatures improved classification performance and presented a comprehensive view of cancer progression. Further examination of these signatures discovered well known and less studied gene and isoform candidates to predict clinically aggressive cancers.

\section{Methods}

\section{RNA-seq data analysis of KIRC}

Clinical information and expression quantification results of RNA-seq data for kidney renal clear cell carcinoma patients were downloaded from the website of Broad Institute's Genome Data Analysis Center (https:// confluence.broadinstitute.org/display/GDAC/Home, 2013_02_03 stddata Run). In total, there are 480 cancer samples with RNA-seq data, including 234 stage I, 48 stage II, 117 stage III and 81 stage IV patients (Table 1). RSEM is used to estimate gene and isoform expression abundance, which is the estimated fraction of transcripts made up by a given isoform and gene [32,33]. Isoforms with expression larger than 0.001 TPM (transcript per million) in at least half of the stage I or stage IV samples were kept. Limma [35] was applied to identify differentially expressed genes and isoforms between 234 stage I and 81 stage IV patients using the criteria: (1) fold change (FC) $\geq 2$ and (2) FDR $\leq 0.001$ (Benjamini and Hochberg's multiple-test adjustment). When significant changes were detected at both gene and isoform levels, only gene signatures were selected for further analysis.

\section{Classification of cancer stages}

Consensus clustering [36] was used to evaluate the effectiveness of gene and isoform signatures for separating early and late stage cancers. Consensus clustering is a resampling-based method to represent the consensus across multiple runs of a clustering algorithm. Given a data set of patients with a certain number of signatures, we resampled the data, partitioned the resampled data into two clusters, and calculated the classification score for each resampled dataset based on the agreement of the clusters with known stages. We defined the classification stability score (SS) as a properly normalized sum of the classification scores of all the resampled datasets (Eq.1). In the equation, the consensus matrix $\mathrm{M}(\mathrm{i}, \mathrm{j})$ is the portion of the resampled dataset $\left\{D^{(h)}: h=1,2, \ldots, H\right\}$ in which two patients $i$ and $j$ are clustered together, $s_{i}$ and $s_{j}$ are the known stages of patients $i$ and $j$, and ES is the expected stability score of the perfect clustering where the entry in consensus matrix $M$ equals 1 for patient pairs with the same stage and the entry equals 0 for patient pairs with different stages. We have 234 stage I and 81 stage IV patients, thus the expected score of the perfect clustering is 30501 . The stability score estimates how sensitive the clustering results are to patient variability and indicates the classification performance to unknown samples. Here we used ConsensusClusterPlus package [37] to subsample signatures and patients 500 times, whereby a subset of gene/isoform signatures and patients (80\%) was sampled without replacement from the original dataset. We implemented

Table 1 Characteristics of patients with RNA-seq data for kidney renal clear cell carcinoma

\begin{tabular}{ccccc}
\hline & Stage I $(\mathbf{n}=\mathbf{2 3 4})$ & Stage II $(\mathbf{n}=\mathbf{4 8})$ & Stage III $(\mathbf{n}=\mathbf{1 1 7})$ & Stage IV $(\mathbf{n}=\mathbf{8 1})$ \\
\hline Age, years, mean \pm SD & $59.9 \pm 12.8$ & $58.4 \pm 12.0$ & $62.9 \pm 12.1$ & $60.8 \pm 9.9$ \\
Gender, Male, n (\%) & $145(62.0 \%)$ & $36(75.0 \%)$ & $76(65.0 \%)$ & $56(69.1 \%)$ \\
Median follow-up, month (minimum - maximum) & $37.8(0.1-112.6)$ & $47.7(0.1-94.3)$ & $29.5(0.1-96.0)$ & $18.9(0.1-87.0)$ \\
No. of deaths (\%) & $38(16.2 \%)$ & $8(16.7 \%)$ & $45(38.5 \%)$ & $64(79.0 \%)$ \\
\hline
\end{tabular}


both hierarchical and kmeans clustering algorithms based on spearman correlation and the stability score of each algorithm was reported separately.

$$
\begin{aligned}
& M(i, j)=\frac{\sum_{h} M^{(h)}(i, j)}{\sum_{h} I^{(h)}(i, j)} \\
& \frac{\sum_{\mathrm{i}, \mathrm{j}, \mathrm{i}<\mathrm{j}} \mathrm{A}_{\mathrm{ij}} \mathrm{M}(\mathrm{i}, \mathrm{j})}{\mathrm{ES}}\left\{\begin{array}{c}
\mathrm{A}_{\mathrm{ij}}=1, \text { if } \mathrm{s}_{\mathrm{i}}=\mathrm{s}_{\mathrm{j}} \\
\mathrm{A}_{\mathrm{ij}}=-1, \text { if } \mathrm{s}_{\mathrm{i}} \neq \mathrm{s}_{\mathrm{j}}
\end{array}\right. \\
& E S=\sum_{i, j, i<j} A_{i j} M(i, j)\left\{\begin{array}{c}
A_{i j}=1 \text { and } M(i, j)=1, \text { if } s_{i}=s_{j} \\
A_{i j}=-1 \text { and } M(i, j)=0, \text { if } s_{i} \neq s_{j}
\end{array}\right.
\end{aligned}
$$

\section{Function enrichment}

Isoform names were converted into gene symbols by UCSC Genome Table Browser (http://genome.ucsc.edu/ cgi-bin/hgTables). Functional enrichment analysis on the list of gene and isoform signatures was implemented in GO biological process as well as KEGG pathways by WebGestalt [38] (http://bioinfo.vanderbilt.edu/webgestalt/). Enrichment p-values were generated by a hypergeometric test and adjusted by Benjamini and Hochberg's multiple-test. Functional categories with FDR $\leq 0.05$ were selected.

\section{Survival analysis}

165 stage II and stage III patients were used as an independent dataset to evaluate whether gene and isoform expression signatures were predictive of increased risk of cancer death by a Cox proportional hazard (PH) model. The patients were divided into two groups according to the median expression value of a given gene and isoform. Survival analysis was performed between higher- and lower-than-median groups. Genes and isoforms with $\mathrm{FDR} \leq 0.05$ were considered to be significantly associated with clinical outcome. A multivariate model adjusting for age and gender of patients was also performed for selected genes and isoforms.

\section{Results}

\section{Isoform profiles provide additional information}

We estimated the alternative splicing activity and found that about $70 \%$ of multi-exon genes expressed two or more isoforms in each sample. This is consistent with the estimate by Griffith et al.[25], which reported $68 \%$ of multi-exon genes showed evidence for expression of multiple isoforms. We considered the isoform with the highest abundance as the "major" isoform and calculated the relative abundance ratio of the "major" isoform to the corresponding gene. For all genes, about $62 \%$ of the major isoforms had a ratio greater than 0.8 (Additional File 1A, the mean of ratio was 0.82 , Median $=0.93, \mathrm{SD}=0.21$ ).
For genes with multiple isoforms, about $40 \%$ of major isoforms had a ratio greater than 0.8 (Additional File 1B, the mean of ratio was 0.71 , Median $=0.72$; $\mathrm{SD}=0.20)$. These results indicate that one isoform is more highly expressed than the others for most genes.

To compare the capacities of gene and isoform expression profiles to detect alternations, we calculated the fold change-based correlation between genes and their major isoforms. The correlation was high for all genes (Figure $1 \mathrm{~A}, \mathrm{R}^{2}=0.64, \mathrm{p}<2.2 \mathrm{e}-16$ ) and even higher if only differentially expressed genes $(\mathrm{FC} \geq 2$ \& $\mathrm{FDR} \leq 0.001$ ) were considered (Figure $1 \mathrm{~B}, \mathrm{R}^{2}=0.89$, $\mathrm{p}<2.2 \mathrm{e}-16$ ), suggesting genes and their major isoforms are quite consistent in capturing expression changes. In contrast, the correlation of differentially expressed isoforms $(F C \geq 2 \& F D R \leq 0.001)$ and their corresponding genes was lower (Figure $1 C, R^{2}=0.35, p<2.2 e-16$ ), which suggests isoform expression profiling provides additional information that cannot be detected at the gene level. This is possibly due to two reasons. One reason may be that isoform switching induces differential splice variants without gene-level expression changes; the other reason is that, with only one isoform altered, the addition of other isoforms to the total gene expression level simply obscures gene-level expression change.

In total, 567 genes showed significant expression changes between stage I and stage IV patients (FC $\geq 2$ \& $\mathrm{FDR} \leq 0.001$, Additional File 2). Interestingly, more genes (1637 vs. 567) were detected significant at the isoform level than the gene level (Additional File 3, Figure 1D). Among the 567 gene signatures, 325 genes (57\%) had at least one isoform with significant expression change (Figure 1D). In contrast, only $20 \%$ of genes with significantly changed isoforms could be detected at the gene level. The remaining $80 \%$ of the genes with significant isoforms did not show significant changes at the gene level, which represents the unique information provided by isoform expression profiles.

For most genes with significantly changed isoforms, only one isoform was altered between early and late stage cancers. Notably, there were only 17 genes with two or more isoforms showing opposite expression changes, leading to no expression changes at the gene level. In these cases, isoform switching mainly contributes to isoform expression alternations. Among the 17 genes, half of them have been reported to be associated with cell proliferation or cancer progression (Additional File 4).

\section{Combining gene and isoform signatures improves cancer stages classification}

Having identified stage-dependent gene and isoform expression signatures, one of the important questions is to assess the power of these signatures to classify 


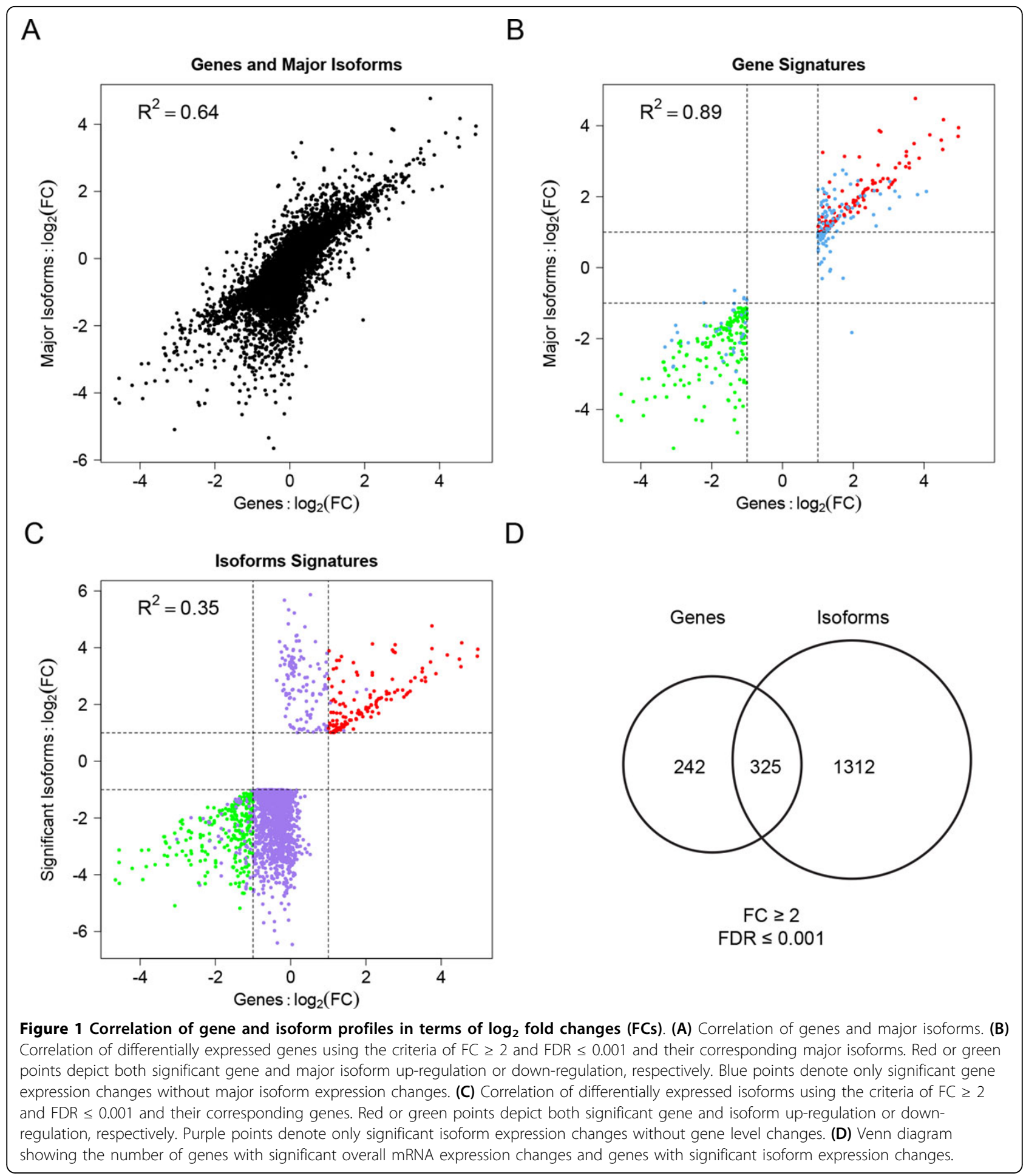

unknown samples, which is essential for early cancer diagnosis. We applied consensus clustering [36], a resampling-based method to estimate classification stability and classification accuracy (See Methods for details). We selected the same number of top-ranked signatures from genes, isoforms, and combined profiles (combining gene and isoforms signatures together) to assess how useful these signatures would be for correctly separating patients with different stages. We used agglomerative hierarchical and k-means methods to implement consensus clustering. The results are similar (Figure 2). Overall, better performance was achieved 


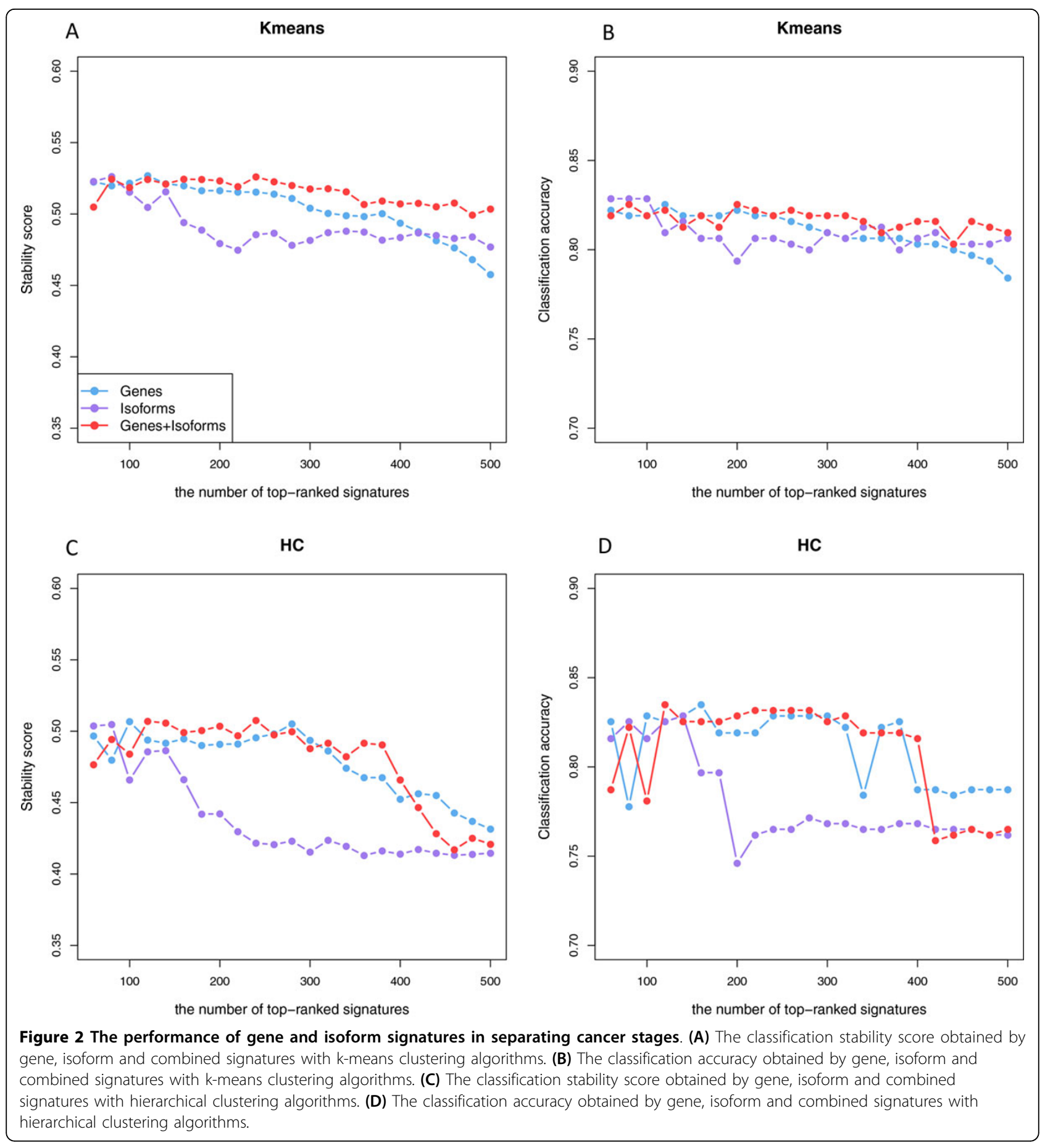

with combined gene and isoform signatures than using gene and isoform signatures alone. The performance using isoform signatures deteriorated quickly with the increasing number of signatures. When the number of signatures increased from 140 to 220, for example, the classification stability score dropped from 0.52 to 0.47 and the number of misclassified patients increased from 57 (accuracy: 81.6\%) to 63 (accuracy: 80.0\%) using k-means based consensus clustering (Figure 2A). With hierarchical clustering, the classification stability score dropped from 0.49 to 0.43 and the number of misclassified patients increases from 54 (accuracy: 82.9\%) to 75 (accuracy: 77.1\%) (Figure 2B). In contrast, the performance using gene and combined signatures was more robust to the number of signatures used. These results suggest that isoform signatures are useful for separating cancer stages, but we should be 
careful about combining isoform information since more uninformative variables or noise would be introduced at such a high resolution level.

\section{Combining gene and isoform signatures provides biological meaningful results}

Gene and isoform signatures associated with cancer stages were interpreted in GO biological process context as well as in KEGG pathway context (Figure 3, Additional File 5). A number of pathways involved in tumor growth, invasion, and metastasis were enriched in both gene and isoform signatures, which included cytokinecytokine receptor interaction, PPAR signaling pathway, p53 signaling pathway, Calcium signaling pathway, etc. (Additional File 5). Cytokines and cytokine receptors are well known to be important contributors to cancer development and progression [39-42]. PPAR signaling is responsible for the regulation of cellular events that range from glucose and lipid homeostasis to cell differentiation and apoptosis, and there is emerging evidence indicating its anti-proliferative actions or tumor promoting effects [43]. Deregulation of calcium signaling is regarded as the primary event in the pathogenesis, growth, invasion, and secondary spread of cancer [44]. As an example, ITPKA was up-regulated in stage IV patients at both gene $\left(\log _{2} \mathrm{FC}=2.14, \mathrm{FDR}=1.8 \mathrm{e}-10\right)$ and isoform levels (uc001znz.2, $\log _{2} \mathrm{FC}=1.99$, FDR $=$ 1.1e-09). High expression of ITPKA has been reported to promote migration of tumor cells by two different mechanisms: ITPKA increases calcium entry that directly influences cell migration in EGF stimulated cells. In growth factor poor medium, ITPKA induces the formation of large cellular protrusions by stabilizing and bundling actin filaments [45].

In addition, there were important biological pathways uniquely identified by gene or isoform signatures. Cell cycle $(F D R=4.0 \mathrm{e}-04)$, cell-cell signaling $(F D R=3.0 \mathrm{e}-$ $04)$, regulation of cell proliferation $(F D R=0.005)$, and $\mathrm{T}$ cell receptor signaling pathways $(F D R=0.05)$ were only observed by gene signatures, which are also known to be associated with tumor progression. For example, the overall mRNA of FOXA1 was highly expressed in stage IV patients $\left(\log _{2} \mathrm{FC}=3.03\right.$, FDR $\left.=2.6 \mathrm{e}-04\right)$. FOXA1 is involved in cell-cell signaling, and it promotes tumor progression in prostate cancer [46,47]. Adherens and tight junctions were only enriched in isoform signatures $(\mathrm{FDR}=2.9 \mathrm{e}-06, \mathrm{FDR}=6.7 \mathrm{e}-05)$. Adherens junction is involved in establishing and maintaining cell-cell adhesion, and disruption of adherens junctions promotes tumor cell invasion and metastasis [48-50]. Tight junction

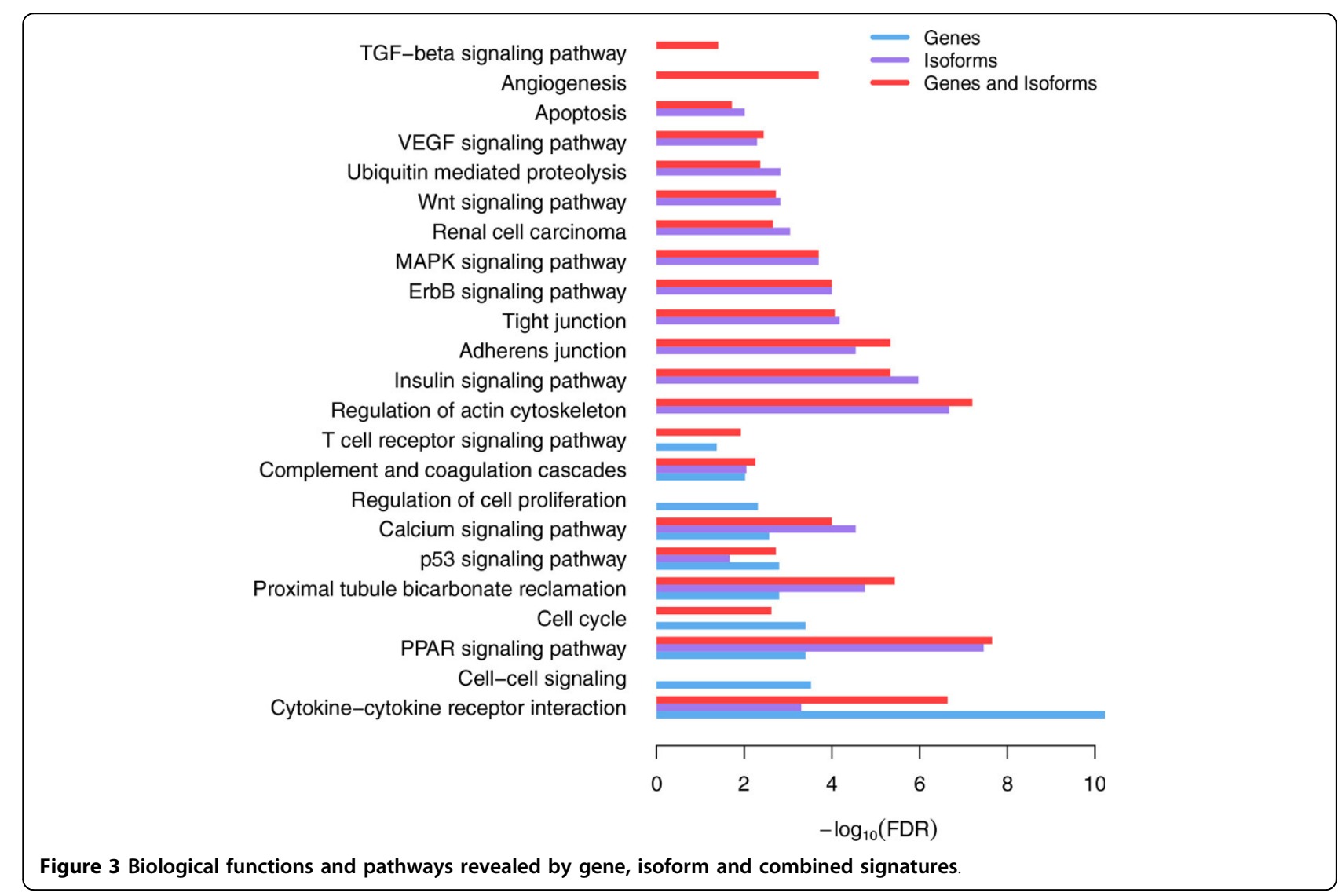


is critical for maintaining cell to cell integrity and the loss of cohesion of the structure will lead to invasion and metastasis of cancer cells[51,52]. Besides, a number of signaling pathways well known to play a crucial role in cancer progression were only observed in isoform signatures, including ErbB signaling pathway, MAPK signaling pathway, Insulin signaling pathway, Wnt signaling pathway, VEGF signaling pathway, etc. These results suggest that isoform signatures provide additional insight into the biological mechanisms related to the tumor progression. The tight junction gene TJB2, for example, showed differential expression only at the isoform level (uc011lrs.2, $\log _{2} \mathrm{FC}=-2.46$, FDR $=4.5 \mathrm{e}-06$; uc011lrt. $1, \log _{2} \mathrm{FC}=-2.34$, $\mathrm{FDR}=1.8-04)$. TJP2 is a candidate tumor suppressor [53] and overexpression of TJP2 will block the cell cycle and inhibit cell proliferation [54].

Notably, combing gene and isoform signatures not only uncovered most of the biological processes detected by gene or isoform profiles but also suggested two additional critical pathways associated with cancer progression, angiogenesis $(\mathrm{FDR}=2.0 \mathrm{e}-04)$ and TGF-beta signaling pathway $(\mathrm{FDR}=0.04)$. Angiogenesis, the process of forming new blood vessels, allows cancer cells to make their own blood supply to obtain oxygen and nutrients, which leads to growth and metastasis [55-57]. The expression of 69 genes involved in angiogenesis was significantly changed at gene and/or isoform levels. 8 genes involved in the TGF-beta signaling pathway showed expression alternations at gene and/or isoform level (Additional File 5).

\section{Gene and isoform signatures predictive with clinical outcome}

We used a Cox proportional hazard (PH) model to evaluate whether the detected gene and isoform expression signatures are predictive of the risk of cancer death. The 165 patients in stage II and stage III of KIRC were taken as an independent dataset and segregated into higher- and lower-than-median groups based on the expression level of the selected gene or isoform. Survival analysis was performed between these two groups. As a result, the expression level of 39 genes and 92 isoforms was found to be significantly associated with survival time (FDR $\leq 0.05)$. The 39 genes included ITPKA and RYR2 (calcium signaling), ITGA8 (regulation of actin cytoskeleton), FOXA1 and ACTN2 (cell-cell signaling), NPR3 (cell proliferation), etc. (Additional File 6). The 92 isoforms, corresponding to 86 genes, contained ITPKA (calcium signaling), ITGA8 (regulation of actin cytoskeleton), TJP2 (tight junction) and ACVR2A (cytokinecytokine receptor interaction), AMOT and BAI1 (angiogenesis), etc. (Additional File 7). Most of these genes have been reported to be involved in cancer progress and metastasis in previous studies.

There were 8 genes whose overall mRNA and isoform expressions were both associated with clinical outcome, including ITPKA, ITGA8, OTOF, ZIC2, COL7A1, CILP, WDR72 and FLRT3. In these cases, the functional isoform dominated the gene expression, and thus a similar signal was obtained at both levels. Consistent with genelevel expression changes, for example, uc001znz.2, the major isoform of ITPKA (calcium signaling) was significantly up-regulated in the stage IV patients $\left(\log _{2} \mathrm{FC}=\right.$ 1.99 , FDR $=1.1 \mathrm{e}-09)$. In Kaplan-Meier estimates, patients with higher ITPKA expression in either isoform or gene level showed lower survival rates (Figures 4A and $4 \mathrm{~B}$ ). The median survival time was 94.3 months versus 47.2 months at both gene level and isoform level. In the univariate Cox $\mathrm{PH}$ model, the hazard ratios for
A

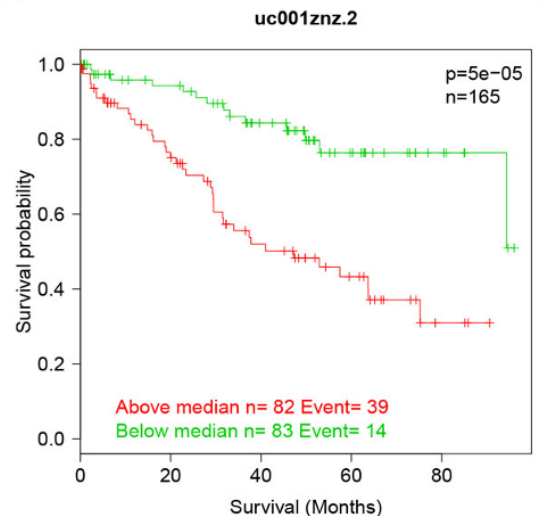

B

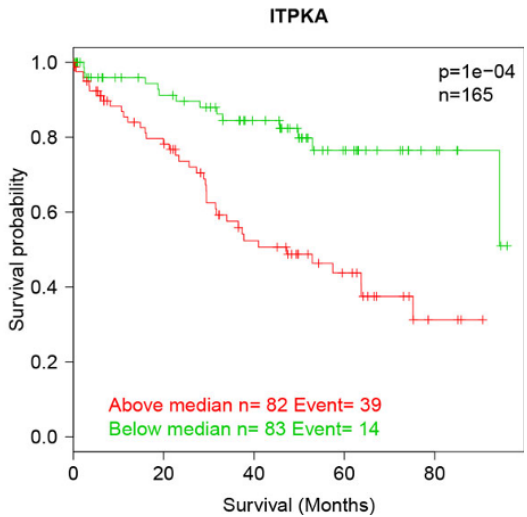

Figure 4 The isoform (uc001znz.2) and gene expression of ITPKA are both predictive of survival in 165 stage II and III patients with KIRC. Expression value which is lower than median is denoted in green and higher than median is noted in red. Patients with low expression value show a significantly higher median survival time compared with patients with high expression value ( $p=5.0$ - 05 for the isoform uc001znz.2 and $p=1.0$ e-04 for ITPKA). 
ITPKA expression above median were $3.46(\mathrm{p}=1 \mathrm{e}-04)$ at gene level and $3.67(\mathrm{p}=5 \mathrm{e}-05)$ at isoform level. Multivariate Cox $\mathrm{PH}$ model analysis adjusting for age and gender was also performed, and ITPKA was also found to be significantly associated with survival time ( $\mathrm{p}=$ 0.0005 at gene level and 0.0002 at isoform level). As we mentioned earlier, ITPKA is a cell motility-promoting protein that increases the metastatic potential of tumor cells. The expression of genes and isoforms associated with cancer stage and clinical outcome make ITPKA the potential target of advanced stage KIRC therapy.

In some cases, however, background expression of nonfunctional isoforms added noise to gene abundance measurements and obscured the gene-level signal. Therefore, only the signal of functional isoforms could be observed. As an example, ubiquitin carboxyl-terminal hydrolase 19 (USP19), a deubiquitinating enzyme that regulates the degradation of various proteins and plays a role in cell proliferation and apoptosis, showed no significant difference on the overall mRNA expression between Stage I and Stage IV patients $(\log 2 \mathrm{FC}=-0.09$, FDR $=0.12$ ). Simultaneously, the overall mRNA expression of USP19 was not significantly associated with survival time (Figure 5D, $\mathrm{p}=0.14$ ). In contrast, uc003cvz.3, the major isoform of USP19 (the relative abundance ratio $=0.4$ ), was significantly down-regulated in stage IV patients $\left(\log _{2} \mathrm{FC}=-1.24\right.$ and $\left.\mathrm{FDR}=0.0003\right)$, and higher uc003cvz.3 expression suggested higher survival rates (Figure 5B). The median survival time for isoform uc003cvz. 3 was 94.3 months versus 49.8 months. In the univariate Cox $\mathrm{PH}$ model, the hazard ratio for uc003cvz.3 expression above the median was $0.37(\mathrm{p}=0.001)$. Multivariate Cox $\mathrm{PH}$ model analysis adjusting for age and gender was also performed and proved that the expression of isoform uc003cvz. 3 was significantly associated with survival time $(\mathrm{p}=0.0005)$. Besides the isoform uc003cvz.3, there was another isoform uc003cwa.2 expressed in similar abundance, which was not significantly changed between stages and was not associated with survival time (Figure 5C). Comparing the structure of these two isoforms, uc003cvz.3 and uc003cwa.2, we found uc003cvz.3 is longer at $\mathrm{N}$ terminal and more functionally important. Isoform uc003cwa. 2 contains only one CS domain, while uc003cvz.3 has two CS domains (Figure 5A), which play an important role in the interaction of USP19 with the cellular inhibitor of apoptosis 2 and influence c-IAP1 and 2 -dependent apoptosis [58]. These results suggest that the expression of the nonfunctional important isoform uc003cwa. 2 obscures the changes of the overall mRNA expression level of UPS19 and that isoform-level analysis is sensitive to detect the signal of functional important isoforms.

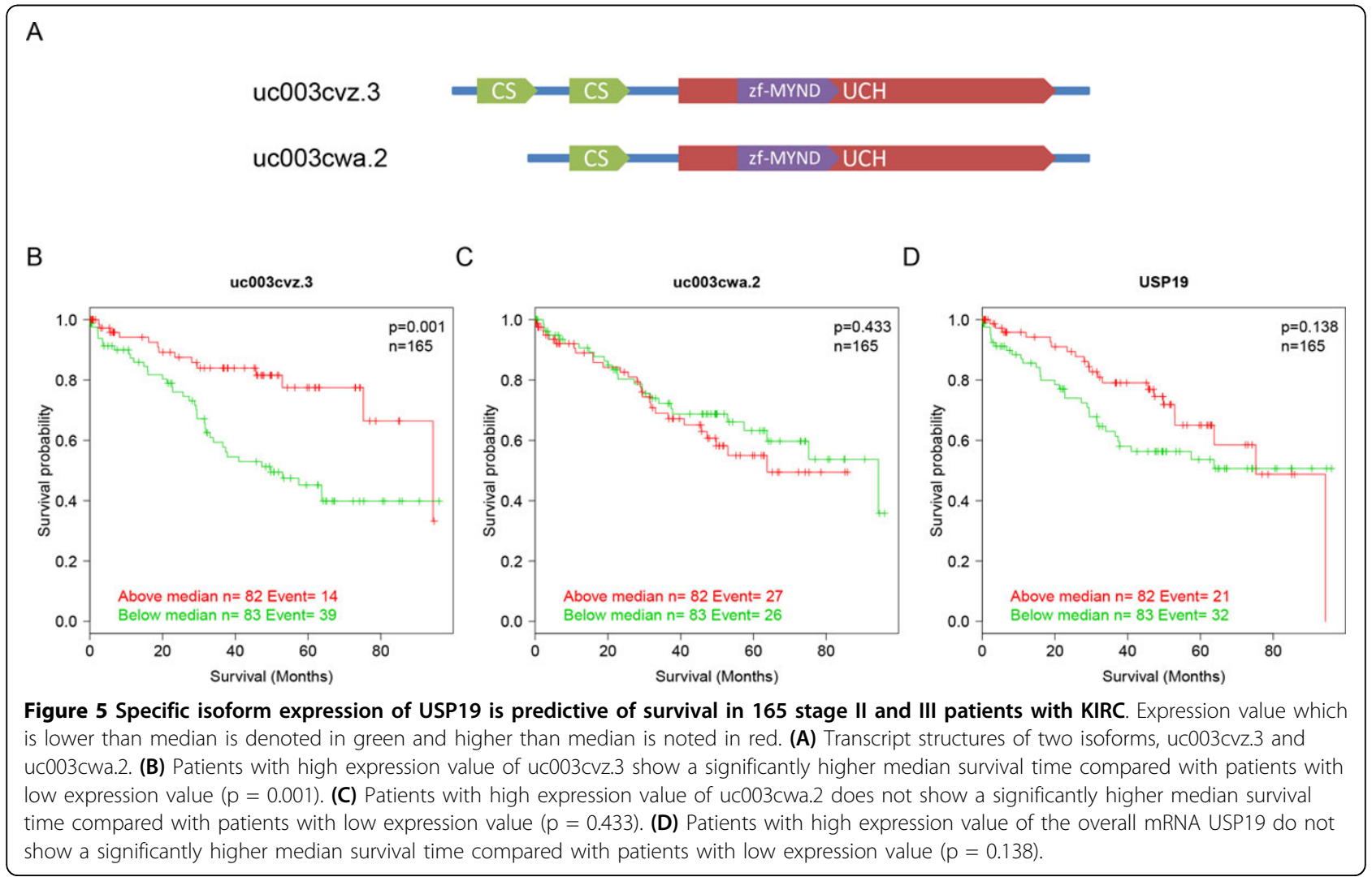




\section{Discussion}

Comparative analysis of expression alternations between early and late stage cancers improves our understanding of cancer growth and metastasis. Previous studies on gene expression profiles have identified overall mRNA expression changes in various kinds of cancers. These overall mRNA transcript level analyses, however, cannot uncover post-transcriptional deregulation and may underestimate the complexity of cancer progression. Recently, post-transcriptional deregulation such as splicing alternations, a key regulatory process by which functionally diverse isoforms can be expressed, has been reported to play an important role in cancer progression. The abundance of each individual isoform, which couples both transcriptional and post-transcriptional regulation, may serve as a valuable source to study the complexity of cancer progression.

RNA-seq technology, enabling a large dynamic range, high resolution, and low technical variance in measuring expression abundance, provides the opportunity of systematically comparing isoform expression profiles between early and late stage cancers. In this study, we not only identified stage-dependent gene and isoform expression signatures, but also compared the usefulness of these two kinds of signatures in terms of separating cancer stages, biological relevance, and prediction of clinical outcome. Remarkably, about $80 \%$ of genes with significant isoform expression changes do not exhibit alternations at the overall mRNA level. These isoforms are useful for separating cancer stages and are enriched in a number of critical biological function and pathways associated with cancer progression and metastasis, such as adherens and tight junctions, ErbB signaling, MAPK signaling, VEGF signaling pathways, etc. Furthermore, the expression abundance of a number of isoforms is significantly associated with the increased risk of death in an independent dataset. These results demonstrate that isoform expression profiling provides unique and important information that cannot be detected by the gene-level. Isoform-level analysis complements the genelevel analysis, and combining gene and isoform signatures improves the classification performance and presents a comprehensive view on the potential biological mechanisms involved in cancer progression.

Moreover, differential expression observed at the isoform level but not at the gene level provides an opportunity for exploring potential post-transcriptional regulatory mechanisms to gain insights into isoform specific regulation. Among 1637 genes with isoform expression changes, only 17 genes contain two or more isoforms showing opposite expression changes, which suggests that isoform switching is not likely to be a major contributor to splicing pattern changes in cancer progression. To find RNA binding proteins responsible for modulating splicing during cancer progression, we can identify stage-dependent splicing pattern changes based on the ratio of alternative spliced isoforms and search for overrepresented nucleotide sequences near stage-associated splicing events. Additionally, analyzing the 3' UTR of genes with differentially expressed isoforms is one way to find the miRNA involved in cancer progression.

Although profiling of individual isoforms provides useful information, we should be careful when we interpret the results from such a high resolution level. Read assignment uncertainty inherent in the RNA-seq data analysis may introduce noise and false positives. Some reads cannot be assigned unequivocally to an isoform since many isoforms share exons. This read assignment uncertainty will affect the accuracy of isoform expression quantification and introduce noise, especially for low abundance genes with multiple isoforms. This is possibly the reason why classification performance drops quickly with the increasing number of isoform expression signatures. On the other hand, many isoforms could be non-functional noise. As a result, the isoforms detected may simply reflect noisy splicing and are not likely to be translated into functional proteins $[59,60]$. For example, one isoform of MLH3 (uc010tuy.1), a DNA mismatch repair gene without significant changes at the overall mRNA level $\left(\log _{2} \mathrm{FC}=-0.04, \mathrm{FDR}=0.6\right)$, was significantly downregulated in the late stage of cancer $\left(\log _{2} \mathrm{FC}=-1.71, \mathrm{FDR}=0.006\right)$. However, this isoform is vulnerable to nonsense-mediated decay and cannot be translated into protein. As another example, one isoform of MGRN1 (uc010uxq.1) with significant expression changes $\left(\log _{2} \mathrm{FC}=2.53\right.$, FDR $\left.=0.001\right)$ was also a noncoding transcript. Consistently, a previous study has reported increased levels of noisy splicing in cancers, leading to marked changes in premature stop-codon frequency for tumor suppressor and oncogenes[60]. Thus it is important to consider splicing noise when identifying stage-dependent isoform expression signatures. To reduce the effect of noisy splicing and read assignment uncertainty, summarizing the reads into more functional important units, e.g., including only reads that map to the coding sequences may be more suited for finding biologically meaningful results.

\section{Conclusion}

Isoform expression profiling extends our knowledge about cancer progression and serves as a useful complement to gene level analysis. Combining gene and isoform expression signatures helps to identify advanced stage cancers and present a comprehensive view on biological mechanisms in cancer development and progression. 


\section{Additional material}

Additional file 1: The distribution of the relative abundance ratio of "major" isoform to the corresponding gene. (A) The ratio distribution for all genes. (B) The ratio distribution for genes with two or more isoforms expressed.

Additional file 2: Differentially expressed genes between the early and the late stages of KIRC (FC $\geq 2$ \& FDR $\leq 0.001)$.

Additional file 3: Differentially expressed isoforms between the early and the late stages of KIRC (FC $\geq 2$ \& FDR $\leq 0.001)$.

Additional file 4: 17 genes have two or more isoforms differentially expressed with opposite change directions

Additional file 5: Functional enrichment results in gene, isoform and combined signatures.

Additional file 6: 39 genes whose expression abundance is predictive of increased risk of cancer death in stage II and III patients

Additional file 7: 92 isoforms whose expression abundance is predictive of increased risk of cancer death in stage II and III patients

\section{Competing interests}

The authors declare that they have no competing interests.

\section{Authors' contributions}

YS led the project and oversaw the analysis. QL and SLZ designed and performed the research. PFS participated in the survival analysis. QL and SLZ wrote the manuscript. All authors have read and approved of the final manuscript.

\section{Acknowledgements}

The authors wish to thank Bjoring Margot for editorial work on this manuscript. This work was supported by National Cancer Institute grants U01 CA163056, P30 CA068485, P50 CA098131, and P50 CA090949 (to YS) QL's work was partially supported by the State Key Program of National Natural Science of China (no. 31230058) and the National Natural Science Foundation of China (no. 31070746)

\section{Declarations}

The publication costs for this article were funded by the corresponding author.

This article has been published as part of BMC Systems Biology Volume 7 Supplement 5, 2013: Selected articles from the International Conference on Intelligent Biology and Medicine (ICIBM 2013): Systems Biology. The full contents of the supplement are available online at http://www biomedcentral.com/bmcsystbiol/supplements/7/S5.

\section{Authors' details}

${ }^{1}$ Center for Quantitative Sciences, Vanderbilt University School of Medicine, Nashville, TN 37232, USA. ²Department of Biomedical Informatics, Vanderbilt University School of Medicine, Nashville, TN 37232, USA. ${ }^{3}$ Department of Statistics, National Cheng Kung University, Taiwan. ${ }^{4}$ Department of Cancer Biology, Vanderbilt University School of Medicine, Nashville, TN 37232, USA. ${ }^{5}$ Department of Biostatistics, Vanderbilt University School of Medicine, Nashville, TN 37232, USA.

Published: 9 December 2013

\section{References}

1. Yokota J: Tumor progression and metastasis. Carcinogenesis 2000, 21(3):497-503.

2. Cui J, Li F, Wang G, Fang X, Puett JD, Xu Y: Gene-expression signatures can distinguish gastric cancer grades and stages. PLoS One 2011, 6(3): e17819.

3. Delgado-Cruzata L, Hruby GW, Gonzalez K, McKiernan J, Benson MC, Santella RM, Shen J: DNA methylation changes correlate with Gleason score and tumor stage in prostate cancer. DNA Cell Biol 2012, 31(2):187-192

4. Dip N, Reis ST, Timoszczuk LS, Viana NI, Piantino CB, Morais DR, Moura CM, Abe DK, Silva IA, Srougi M, et al: Stage, grade and behavior of bladder urothelial carcinoma defined by the microRNA expression profile. J Urol 2012, 188(5):1951-1956

5. Fearon ER, Vogelstein B: A genetic model for colorectal tumorigenesis. Cell 1990, 61(5):759-767.

6. Fransson S, Abel F, Kogner P, Martinsson T, Ejeskar K: Stage-dependent expression of PI3K/Aktpathway genes in neuroblastoma. Int J Oncol 2013, 42(2):609-616.

7. Kinzler KW, Vogelstein B: Lessons from hereditary colorectal cancer. Cell 1996, 87(2):159-170.

8. Oue N, Mitani Y, Motoshita J, Matsumura S, Yoshida K, Kuniyasu H, Nakayama H, Yasui W: Accumulation of DNA methylation is associated with tumor stage in gastric cancer. Cancer 2006, 106(6):1250-1259.

9. Smith JJ, Deane NG, Wu F, Merchant NB, Zhang B, Jiang A, Lu P, Johnson JC, Schmidt C, Bailey CE, et al: Experimentally derived metastasis gene expression profile predicts recurrence and death in patients with colon cancer. Gastroenterology 2010, 138(3):958-968

10. Vogelstein B, Fearon ER, Hamilton SR, Kern SE, Preisinger AC, Leppert M, Nakamura Y, White R, Smits AM, Bos JL: Genetic alterations during colorectal-tumor development. N Engl J Med 1988, 319(9):525-532.

11. Watts GS, Futscher BW, Holtan N, Degeest K, Domann FE, Rose SL: DNA methylation changes in ovarian cancer are cumulative with disease progression and identify tumor stage. BMC Med Genomics 2008, 1:47

12. Mannan A, Hahn-Stromberg V: K-ras mutations are correlated to lymph node metastasis and tumor stage, but not to the growth pattern of colon carcinoma. APMIS 2012, 120(6):459-468.

13. Soong R, Powell B, Elsaleh H, Gnanasampanthan G, Smith DR, Goh HS, Joseph D, lacopetta B: Prognostic significance of TP53 gene mutation in 995 cases of colorectal carcinoma. Influence of tumour site, stage, adjuvant chemotherapy and type of mutation. Eur J Cancer 2000, 36(16):2053-2060.

14. Zhang Q, Ying J, Li J, Fan Y, Poon FF, Ng KM, Tao Q, Jin J: Aberrant promoter methylation of DLEC1, a critical 3p22 tumor suppressor for renal cell carcinoma, is associated with more advanced tumor stage. $J$ Urol 2010, 184(2):731-737.

15. Zhu X, Qin X, Fei M, Hou W, Greshock J, Bachman KE, Kang J, Qin CY: Loss and reduced expression of PTEN correlate with advanced-stage gastric carcinoma. Exp Ther Med 2013, 5(1):57-64.

16. Apostolou P, Toloudi M, Chatziioannou M, loannou E, Papasotiriou I: Cancer stem cells stemness transcription factors expression correlates with breast cancer disease stage. Curr Stem Cell Res Ther 2012, 7(6):415-419.

17. Okamura K, Koike H, Sekine $Y$, Matsui H, Suzuki K: Survivin and its spliced isoform gene expression is associated with proliferation of renal cancer cells and clinical stage of renal cancer. Cancer Epidemio/ 2009, 33(2):137-141.

18. Tomlinson DC, Knowles MA: Altered splicing of FGFR1 is associated with high tumor grade and stage and leads to increased sensitivity to FGF1 in bladder cancer. Am J Pathol 2010, 177(5):2379-2386.

19. Marabese M, Marchini S, Marrazzo E, Mariani P, Cattaneo D, Fossati $R$, Compagnoni A, Signorelli M, Moll UM, Codegoni AM, et al: Expression levels of p53 and p73 isoforms in stage I and stage III ovarian cancer. Eur J Cancer 2008, 44(1):131-141.

20. Terpe HJ, Storkel S, Zimmer U, Anquez V, Fischer C, Pantel K, Gunthert U: Expression of CD44 isoforms in renal cell tumors. Positive correlation to tumor differentiation. Am J Pathol 1996, 148(2):453-463.

21. Zhou YJ, Xie YT, Gu J, Yan L, Guan GX, Liu X: Overexpression of cyclin E isoforms correlates with poor prognosis in rectal cancer. Eur J Surg Oncol 2011, 37(12):1078-1084.

22. Karni-Schmidt O, Castillo-Martin M, Shen TH, Gladoun N, DomingoDomenech J, Sanchez-Carbayo M, Li Y, Lowe S, Prives C, Cordon-Cardo C Distinct expression profiles of p63 variants during urothelial development and bladder cancer progression. Am J Pathol 2011, 178(3):1350-1360.

23. Ghigna C, Valacca C, Biamonti G: Alternative splicing and tumor progression. Curr Genomics 2008, 9(8):556-570

24. Wang Z, Gerstein M, Snyder M: RNA-Seq: a revolutionary tool for transcriptomics. Nat Rev Genet 2009, 10(1):57-63.

25. Griffith M, Griffith OL, Mwenifumbo J, Goya R, Morrissy AS, Morin RD, Corbett $\mathrm{R}$, Tang MJ, Hou YC, Pugh TJ, et al: Alternative expression analysis by RNA sequencing. Nat Methods 2010, 7(10):843-847. 
26. Nicolae M, Mangul S, Zelikovsky A: Estimation of alternative splicing isoform frequencies from RNA-Seq data. Algorithms Mol Biol 2011, 6(1):9.

27. Huang Y, Hu Y, Jones CD, Macleod JN, Chiang DY, Liu Y, Prins JF, Liu J: A Robust Method for Transcript Quantification with RNA-Seq Data. J Comput Biol 2013, 20(3):167-187.

28. Katz Y, Wang ET, Airoldi EM, Burge CB: Analysis and design of RNA sequencing experiments for identifying isoform regulation. Nat Methods 2010, 7(12):1009-1015.

29. Trapnell C, Williams BA, Pertea G, Mortazavi A, Kwan G, van Baren MJ, Salzberg SL, Wold BJ, Pachter L: Transcript assembly and quantification by RNA-Seq reveals unannotated transcripts and isoform switching during cell differentiation. Nat Biotechnol 2010, 28(5):511-515.

30. Trapnell C, Hendrickson DG, Sauvageau M, Goff L, Rinn JL, Pachter L: Differential analysis of gene regulation at transcript resolution with RNAseq. Nat Biotechnol 2013, 31(1):46-53.

31. Mezlini AM, Smith EJ, Fiume M, Buske O, Savich GL, Shah S, Aparicio S, Chiang DY, Goldenberg A, Brudno M: iReckon: Simultaneous isoform discovery and abundance estimation from RNA-seq data. Genome Res 2013, 23(3):519-529.

32. Li B, Dewey CN: RSEM: accurate transcript quantification from RNA-Seq data with or without a reference genome. BMC Bioinformatics 2011, 12:323.

33. Li J, Jiang $H$, Wong $W H$ : Modeling non-uniformity in short-read rates in RNA-Seq data. Genome Biol 2010, 11(5):R50.

34. Garber M, Grabherr MG, Guttman M, Trapnell C: Computational methods for transcriptome annotation and quantification using RNA-seq. Nat Methods 2011, 8(6):469-477.

35. Smyth GK: Linear models and empirical bayes methods for assessing differential expression in microarray experiments. Statistical applications in genetics and molecular biology 2004, 3:Article3.

36. Monti S, Tamayo P, Mesirov J, Golub T: Consensus clustering: A resampling-based method for class discovery and visualization of gene expression microarray data. Mach Learn 2003, 52(1-2):91-118.

37. Wilkerson MD, Hayes DN: ConsensusClusterPlus: a class discovery tool with confidence assessments and item tracking. Bioinformatics 2010 26(12):1572-1573.

38. Zhang B, Kirov S, Snoddy J: WebGestalt: an integrated system for exploring gene sets in various biological contexts. Nucleic Acids Res 2005, 33(Web Server):W741-748

39. Dranoff $\mathrm{G}$ : Cytokines in cancer pathogenesis and cancer therapy. Nat ReV Cancer 2004, 4(1):11-22.

40. Tsujimoto H, Ono S, Ichikura T, Matsumoto $Y$, Yamamoto J, Hase K: Roles of inflammatory cytokines in the progression of gastric cancer: friends or foes? Gastric Cancer 2010, 13(4):212-221

41. Chung YC, Chang YF: Significance of inflammatory cytokines in the progression of colorectal cancer. Hepatogastroenterology 2003, 50(54):1910-1913.

42. Miki S, Iwano M, Miki Y, Yamamoto M, Tang B, Yokokawa K, Sonoda T, Hirano T, Kishimoto T: Interleukin-6 (IL-6) functions as an in vitro autocrine growth factor in renal cell carcinomas. FEBS Lett 1989, 250(2):607-610.

43. Pazienza V, Vinciguerra M, Mazzoccoli G: PPARs Signaling and Cancer in the Gastrointestinal System. PPAR Res 2012, 2012:560846.

44. Capiod T, Shuba Y, Skryma R, Prevarskaya N: Calcium signalling and cance cell growth. Subcell Biochem 2007, 45:405-427

45. Windhorst S, Fliegert R, Blechner C, Mollmann K, Hosseini Z, Gunther T, Eiben M, Chang L, Lin HY, Fanick W, et al: Inositol 1,4,5-trisphosphate 3kinase-A is a new cell motility-promoting protein that increases the metastatic potential of tumor cells by two functional activities. $J$ Biol Chem 2010, 285(8):5541-5554.

46. Gerhardt J, Montani M, Wild P, Beer M, Huber F, Hermanns T, Muntener M, Kristiansen G: FOXA1 promotes tumor progression in prostate cancer and represents a novel hallmark of castration-resistant prostate cancer. Am J Pathol 2012, 180(2):848-861.

47. Sahu B, Laakso M, Ovaska K, Mirtti T, Lundin J, Rannikko A, Sankila A, Turunen JP, Lundin M, Konsti J, et al: Dual role of FoxA1 in androgen receptor binding to chromatin, androgen signalling and prostate cancer. EMBO J 2011, 30(19):3962-3976.

48. Chang YW, Marlin JW, Chance TW, Jakobi R: RhoA mediates cyclooxygenase-2 signaling to disrupt the formation of adherens junctions and increase cell motility. Cancer Res 2006, 66(24):11700-11708.
49. Boissan M, De Wever O, Lizarraga F, Wendum D, Poincloux R, Chignard N, Desbois-Mouthon C, Dufour S, Nawrocki-Raby B, Birembaut $P$, et al: Implication of metastasis suppressor NM23-H1 in maintaining adherens junctions and limiting the invasive potential of human cancer cells. Cancer Res 2010, 70(19):7710-7722.

50. Vasioukhin V: Adherens junctions and cancer. Subcell Biochem 2012, 60:379-414.

51. Martin TA, Mason MD, Jiang WG: Tight junctions in cancer metastasis. Front Biosci 2011, 16:898-936.

52. Martin TA, Jiang WG: Loss of tight junction barrier function and its role in cancer metastasis. Biochim Biophys Acta 2009, 1788(4):872-891.

53. Glaunsinger BA, Weiss RS, Lee SS, Javier R: Link of the unique oncogenic properties of adenovirus type 9 E4-ORF1 to a select interaction with the candidate tumor suppressor protein ZO-2. EMBO J 2001 , 20(20):5578-5586.

54. Gonzalez-Mariscal L, Tapia R, Huerta M, Lopez-Bayghen E: The tight junction protein ZO-2 blocks cell cycle progression and inhibits cyclin D1 expression. Ann N Y Acad Sci 2009, 1165:121-125.

55. Folkman J: Role of angiogenesis in tumor growth and metastasis. Semin Oncol 2002, 29(6 Suppl 16):15-18.

56. Pralhad T, Madhusudan S, Rajendrakumar K: Concept, mechanisms and therapeutics of angiogenesis in cancer and other diseases. J Pharm Pharmacol 2003, 55(8):1045-1053.

57. Carmeliet $P$, Jain RK: Angiogenesis in cancer and other diseases. Nature 2000, 407(6801):249-257.

58. Mei Y, Hahn AA, Hu S, Yang X: The USP19 deubiquitinase regulates the stability of c-IAP1 and c-IAP2. J Biol Chem 2011, 286(41):35380-35387.

59. Pickrell JK, Pai AA, Gilad Y, Pritchard JK: Noisy splicing drives mRNA isoform diversity in human cells. PLoS Genet 2010, 6(12):e1001236.

60. Chen L, Tovar-Corona JM, Urrutia AO: Increased levels of noisy splicing in cancers, but not for oncogene-derived transcripts. Hum Mol Genet 2011, 20(22):4422-4429.

doi:10.1186/1752-0509-7-S5-S7

Cite this article as: Liu et al:: Gene and isoform expression signatures associated with tumor stage in kidney renal clear cell carcinoma. BMC Systems Biology 2013 7(Suppl 5):S7.

\section{Submit your next manuscript to BioMed Central and take full advantage of:}

- Convenient online submission

- Thorough peer review

- No space constraints or color figure charges

- Immediate publication on acceptance

- Inclusion in PubMed, CAS, Scopus and Google Scholar

- Research which is freely available for redistribution
C Biomed Central 